\title{
Generation and characterization of a Myh6-driven Cre knockin mouse line
}

\author{
Xinyan Huang • Lei Yan · Shan Kou • Jufeng Meng • Zhengkai Lu • \\ Chao-Po Lin $\cdot$ Chen Liu $\cdot$ Hui Zhang $(\mathbb{C}$
}

Received: 2 August 2021 / Accepted: 10 September 2021/Published online: 20 September 2021

(C) The Author(s) 2021

\begin{abstract}
Gene deletion by the Cre-Loxp system has facilitated functional studies of many critical genes in mice, offering important insights and allowing deeper understanding on the mechanisms underlying organ development and diseases, such as heart development and diseases. In this study, we generated a Myh6-Cre knockin mouse model by inserting the IRES-Crewpre-polyA cassette between the translational stop codon and the $3^{\prime}$ untranslated region of the endogenous Myh6 gene. By crossing knockin mice with the
\end{abstract}

Xinyan Huang and Lei Yan have contributed equally to this work.

Supplementary Information The online version contains supplementary material available at https://doi.org/10.1007/ s11248-021-00285-4.

X. Huang $\cdot$ L. Yan $\cdot$ S. Kou $\cdot$ J. Meng $\cdot$

Z. Lu $\cdot$ C.-P. Lin $\cdot$ H. Zhang $(\bowtie)$

School of Life Science and Technology, ShanghaiTech

University, 393 Middle Huaxia Road, Pudong,

Shanghai 201210, China

e-mail: zhanghui1@shanghaitech.edu.cn

C. Liu $(\bowtie)$

Department of Cardiac Surgery, Zhongshan Hospital, Fudan University, 1409 Xietu Road, Xuhui,

Shanghai 200032, China

e-mail: liu.chen@zs-hospital.sh.cn

X. Huang $\cdot$ L. Yan $\cdot$ S. Kou $\cdot$ Z. Lu

University of Chinese Academy of Sciences,

Beijing 100049, China
Rosa26 reporter lines, we found that Myh6-Cre targeted cardiomyocytes at the embryonic and postnatal stages. In addition, we were able to inactivate the desmosome gene Desmoplakin (Dsp) by breeding Myh6-Cre mice with a conditional Dsp ${ }^{f l o x}$ knockout mouse line, which resulted in embryonic lethality during the mid-term pregnancy. These results suggest that the new Myh6-Cre mouse line can serve as a robust tool to dissect the distinct roles of genes involved in heart development and function.

Keywords Cre-loxp - Mouse model - Development . Desmoplakin

\section{Introduction}

Congenital heart disease is the most common birth defect in humans and the leading cause of death in the first year of life (Hoffman 1995; Wolf and Basson 2010; Bruneau and Srivastava 2014; Gelb and Chung 2014). Gene deletion by the Cre-Loxp system has facilitated causative studies of many genes in mice that are essential for heart development and function. A variety of transgenic constitutive or inducible Cre mouse tools have been generated and employed in order to manipulate the expression of target genes in cardiomyocytes. However, transgenes may not always correctly reflect the expression of endogenous genes 
(Laboulaye et al. 2018), and the copy number of insertional transgenes may be reduced during passages which directly influences Cre expression levels (Davis et al. 2012). For the inducible CreER or MerCreMer (Cre recombinase fused to two mutated estrogen receptor ligand binding domains), regardless of being transgenic or knockin mouse lines, the inducible Cre is not able to mediate complete excision, and the administration of tamoxifen is toxic to embryos and could easily lead to abortion before sample collection. Furthermore, some transgenic inducible Cre mice displayed cardiac functional defects after tamoxifen treatment (Buerger et al. 2006; Hall et al. 2011; Koitabashi et al. 2009; Hougen et al. 2010; Lexow et al. 2013; Bersell et al. 2013; Molkentin and Robbins 2009), which may lead to the misinterpretation of data in different studies.

Myh6, also known as $\alpha M H C$, Myhc and Myhca, encodes the cardiac muscle specific protein, alphamyosin heavy chain, which is dynamically expressed in cardiomyocytes and with significant importance for heart development (Carniel et al. 2005; Ching et al. 2005; Posch et al. 2011; Ng et al. 1991). Mutations in MYH7 in humans cause dilated and hypertrophic cardiomyopathy (Carniel et al. 2005; Richard et al. 2003). MYH6 is expressed at low levels in adult human cardiomyocytes and the role of genetic variants in MYH6 in human diseases is uncertain. In this study, we created a Myh6-Cre knockin mouse line by inserting the IRES-Cre-wpre-polyA cassette between the translational stop codon and the $3^{\prime}$ untranslated region (UTR) of the endogenous Myh6 gene. By immunostaining experiments, we found that Myh6Cre targeted cardiomyocytes during fetal and postnatal stages. In addition, we were able to efficiently delete the desmosome gene Desmoplakin (Dsp) from the mouse heart using Myh6-Cre, which resulted in embryonic lethality during mid-term pregnancy. Our results showed that Myh6-Cre is a useful genetic tool that enables the deletion of target genes in cardiomyocytes.

\section{Materials and methods}

Animals

All mouse studies were performed in accordance with the guidelines provided by the institutional Animal
Care and Use Committee at ShanghaiTech University. All mice were maintained in specific pathogen-free conditions. Mice were bred with a normal diet and maintained on a C57BL6/J/ICR background. Both male and female animals were used in the analyses. R26-tdTomato and Dsp $p^{f l o x}$ mice have been previously reported (Madisen et al. 2010; Vasioukhin et al. 2001). The R26-tdTomato mouse line was kindly provided by the Shanghai Model Organisms Center, INC. The $D s p^{f l o x}$ mouse line was purchased from the Jackson Laboratory. For generation of the Myh6-Cre mouse line, the CRISPR/Cas9 technology was used to insert the internal ribosome entry site (IRES)-Cre cassette, the woodchuck hepatitis virus posttranscriptional regulatory element (wpre), and a polyA sequence between the translational stop codon and the $3^{\prime}$ UTR of the endogenous Myh6 gene. The Myh6-Cre mouse line was generated by the Shanghai Model Organisms Center, INC. This newly generated mouse line is available from the corresponding author with a completed material transfer agreement.

\section{Genotyping}

Genomic DNA was extracted from either the embryonic yolk sac or the mouse tail. The harvested tissues were lysed through incubation with proteinase $\mathrm{K}$ for a total of $12 \mathrm{~h}$ at a temperature of $55^{\circ} \mathrm{C}$, followed by centrifugation for $8 \mathrm{~min}$. This process allowed us to obtain a supernatant containing the genomic DNA, which was precipitated by adding isopropanol. All embryos and mice used in our experiments were genotyped with specific primers that distinguished the knockin from the wild-type alleles. The genotyping primers used in the Myh6-Cre mouse line were: $5^{\prime}$ TTCCCAAGGGCATTTTATTAG- $3^{\prime}$ and $5^{\prime}$ CTTTGGGCTTGGCATCATCTGGT- $3^{\prime}$ (wild type allele); 5'-CAGAAGATGCACGACGAG-3' and 5'CAGCCCCTTGTTGAATACG-3' (knock-in allele).

\section{Immunostaining}

We performed immunofluorescence staining according to previously described protocols (Huang et al. 2019). Briefly, mouse embryos or hearts were collected and fixed in $4 \%$ paraformaldehyde (PFA) for a total of $30 \mathrm{~min}$ to $1 \mathrm{~h}$, depending on the age of the tissues. After three consecutive washes in PBS, mouse embryos or hearts were photographed using 
fluorescence microscopy (Olympus, MVX10). The tissues were then dehydrated in $30 \%$ sucrose and embedded in optimum cutting temperature (OCT) compound (Sakura). Cryosections collected with $9-\mu \mathrm{m}$ thickness were air-dried and then blocked with blocking buffer (5\% donkey serum, $0.1 \%$ Triton $\mathrm{X}-100$ in PBS). Both of these steps were performed for $30 \mathrm{~min}$ each at room temperature. Primary antibodies were incubated overnight at $4{ }^{\circ} \mathrm{C}$. The following primary antibodies were used: tdTomato (Rockland, 600-4,010,379, 1:1000; Sicgen, AB8181200, 1:1000), PDGFRa (R\&D, AF1062, 1:500), PDGFRb (eBioscience, 14-1402-82, 1:500), aSMA (Sigma, F3777, 1:400), CDH5 (R\&D, AF1002, 1:200), TNNI3 (Abcam, ab56357, 1:200), Cre (Millipore, MAB3120, 1:200), MYH6 (Invitrogen, PA597,224,1:200) and WT1 (Abcam, ab89901, 1:100). Signals were developed with Alexa fluorescence antibodies (Invitrogen). The images obtained were acquired using a confocal microscope (Nikon A1R).

\section{Real-time quantitative PCR}

Mouse embryos at embryonic day 10.5 or adult hearts at postnatal 8 weeks were harvested. The hearts or non-cardiac tissues were treated with Trizol in order to extract RNA in accordance with the manufacturer's instructions (Invitrogen). We converted the RNA to cDNA using the Prime Script RT kit (Vazyme). We used the SYBR Green qPCR master mix (Vazyme) and amplified the cDNA on a StepOnePlusTM realtime PCR system (Applied Biosystems). The primers used to detect the mRNA levels of the Dsp, Myh6 and Gapdh are listed below. Dsp: 5'-AAACCGGCACCATGTCTAGA-3 $3^{\prime}$ and $5^{\prime}-$ CTCCGAATTTCAGTTCCGGC-3'; Myh6: $3^{\prime}$ CAATGCAGAGTCGGTGAAGG-3' and $5^{\prime}$ CCTCTGTCTGGTAGGTGAGC-3'; Gapdh: 5'TTGTCTCCTGCGACTTCAAC-3' and 5'-GTCATACCAGGAAATGAGCTTG- $3^{\prime}$.

\section{Hematoxylin and Eosin staining}

Mouse hearts at postnatal 8 weeks were fixed in $4 \%$ PFA at $4{ }^{\circ} \mathrm{C}$ for $1 \mathrm{~h}$ and then dehydrated in $30 \%$ sucrose overnight. The next day, the hearts were processed into OCT-embedded serial sections. Slides were incubated in hematoxylin solution for 8-10 $\mathrm{min}$ and then rinsed in running tap water. The slides were then treated with $1 \%$ concentrated hydrochloric acid in $70 \%$ ethanol for $1.5 \mathrm{~min}$ and rinsed in running tap water. After soaking in PBS for 3 min and washed by running tap water, the slides were stained with eosin solution for 3-5 min followed by dehydration in ethanol and xylene. Images were acquired by stereomicroscope (Olympus MVX10).

\section{Echocardiographic assessment}

Adult mice at postnatal 8 weeks or 4 months were anesthetized with inhalation of isoflurane (2.5\%-3\%) and heart rate was maintained at 400-500 bpm. The chest was shaved and further cleaned with hair removal gel cream. Mice were placed on a warm board in the supine position with the limbs taped onto the metal electrocardiographic leads. Acoustic gel was applied to the thorax surface as coupling medium. Using a VisualSonics Vevo 2100 system and a 30-MHz microvisualization scan head probe, M-mode images and real-time B-mode cine loops of the left ventricle were acquired for cardiac structure and function assessment. Using the long-axis view, left ventricular end-systolic volume and end-diastolic volume, as well as the ejection fraction were calculated by identifying frames with the maximal and minimal cross-sectional area and width. All data were presented as mean values \pm SEM. The echocardiographers were blinded to animal group assignments.

\section{Cardiomyocyte isolation}

Myh6-Crel $+;$ R26-tdTomato/ + mice at postnatal 8 weeks were intraperitoneally injected with about 150 USP units heparin $15 \mathrm{~min}$ in advance. Then mice were intraperitoneally injected with $10 \%$ chloral hydrate. The dissected hearts were perfused with modified Tyrode's solution (MTS) for 5 min. Later, the hearts were perfused with MTS containing $1 \mathrm{mg} /$ $\mathrm{ml}$ collagenase II and $0.08 \mathrm{mg} / \mathrm{ml}$ Protease XIV. The digested hearts were shredded with forceps and filtered through a $70 \mu \mathrm{m}$ strainer. The filtered cells were centrifuged at $20 \times \mathrm{g}$ for $3 \mathrm{~min}$ at $4{ }^{\circ} \mathrm{C}$ and most of the cardiomyocytes were pelleted. The pellets were resuspended in MTS with $0.5 \%$ BSA and then centrifuged at $100 \times \mathrm{g}$ for $5 \mathrm{~min}$ at $4{ }^{\circ} \mathrm{C}$ in Percoll to remove the dead cells. The cardiomyocytes were resuspended in MTS with $0.5 \%$ BSA and implanted in 
24-well plates. Images were taken under a stereo microscope (Olympus MVX10).

\section{Western blot}

The hearts at postnatal 8 weeks were dissected and washed in PBS buffer. Then about $15 \mathrm{mg}$ tissues were lysed in $300 \mu \mathrm{L}$ RIPA lysis buffer and incubated on ice for $30 \mathrm{~min}$. All protein samples were mixed with $5 \times$ loading buffer and boiled at $95{ }^{\circ} \mathrm{C}$ for $5 \mathrm{~min}$. The lysates were analyzed by SDS/PAGE and transferred onto the polyvinylidene fluoride membrane. Membranes were blocked for $1 \mathrm{~h}$ at room temperature by using 5\% BSA-TBST solution. Then the membranes were incubated with primary antibodies Anti-MYH6 (Invitrogen, PA5-97,224, 1:1000), Anti-Cre recombinase (Millipore, MAB3120, 1:1000) at $4{ }^{\circ} \mathrm{C}$ overnight. The next day, after washing primary antibodies with TBST, membranes were incubated with HRPconjugated secondary antibodies at room temperature for $1 \mathrm{~h}$. HRP-anti- $\beta$-Actin (ZENBIO, 700068, $1: 10000)$ primary antibodies were just incubated at room temperature for $1 \mathrm{~h}$. Signals were revealed with the enhanced chemiluminescence kit.

\section{Statistics analysis}

All data were representative of at least three independent experiments and presented as mean values \pm SEM. Data of the two groups were analyzed using an unpaired Student's t-test, and comparison between more than two groups was performed using analysis of variance (ANOVA), followed by Tukey's multiple comparison test. $p<0.05$ indicated statistical significance.

\section{Results}

Generation of Myh6-Cre mice

Myh6 mutations lead to dilated and hypertrophic cardiomyopathy, as well as atrial septal defect (Posch et al. 2011; Ching et al. 2005; Carniel et al. 2005). In order to avoid mutating or disrupting Myh6, we generated a knockin mouse line Myh6-Cre using CRISPR/Cas9 to insert the IRES-Cre-wpre-polyA cassette between the translational stop codon and the $3^{\prime}$ UTR of the endogenous Myh6 gene (Fig. 1a).
Fig. 1 Strategy for generating Myh6-Cre mice. a The strategy for generating Myh6-Cre mice. The IRES-Cre-wpre-polyA cassette was inserted between the translational stop codon and the 3'UTR. b PCR results show that homologous recombination was detected in the F0 generation mice. c Locations of the genotyping primers and an example of genotyping result showing the PCR products

Specifically, we injected the Cas9 mRNA, gRNA ( $5^{\prime}$ acagcgagggtctgctggag- $3^{\prime}$ ), and the donor targeting vector, which contains $5^{\prime}$ homologous arm $(2.5 \mathrm{~kb})$, IRES-Cre-wpre-poly A cassette, and $3^{\prime}$ homologous arm $(4.0 \mathrm{~kb})$, into C57BL6/J zygotes, which were subsequently transferred into pseudo-pregnant mice. With 21 offspring of F0 generation obtained, we designed four primers (Primers I and II to amplify the wild type allele $[10.5 \mathrm{~kb}]$ or the recombined $5^{\prime}$ arm [6.8 kb]; Primers III and IV to amplify the recombined $3^{\prime}$ arm [4.8 kb]) to screen the F0 mice, and finally found 3 founders with successful homologous recombination (Fig. 1a, b). We next sequenced the PCR products to further confirm the correct recombination, and then crossed the 3 founders with wild type mice to get $\mathrm{F} 1$ generation.

To optimize the genotyping protocol for Myh6-Cre knockin, we re-designed four PCR primers (P1 and P2 to amplify the wild type allele [923 bp] and P3 and P4 to amplify the inserted site [452 bp]), which were located outside or inside of the IRES-Cre-wpre-polyA cassette sequence (Fig. 1c). Genotyping by PCR using the forward and reverse primers showed that the IRESCre-wpre-polyA cassette was successfully inserted into the Myh6 locus (Fig. 1c).

Myh6-Cre labels cardiomyocytes from embryonic stage to adulthood

To characterize the Myh6-Cre mouse line, we subsequently crossed the Myh6-Cre/ + mice with a reporter line containing a stop cassette flanked by loxp sites upstream of tdTomato at the Rosa26 locus (Rosa26loxp-stop-loxp-tdTomato, or R26-tdTomato)(Madisen et al. 2010). This crossing allowed us to generate a Myh6-Crel + ;R26-tdTomato/ + mouse line. Importantly, the loxp-flanked stop cassette, which prevents the transcription of the downstream tdTomato, was thus deleted in the Cre-expressing cells, leading to the expression of tdTomato. 
(a)
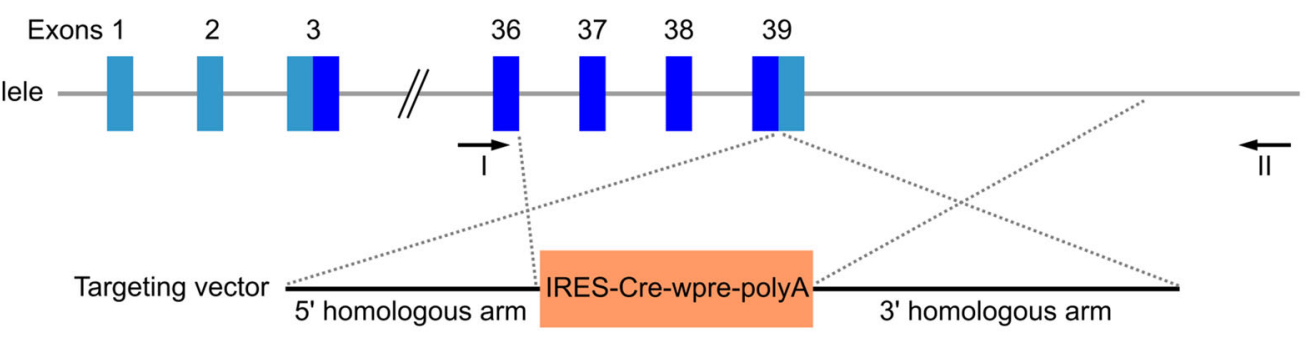

Wild type allele

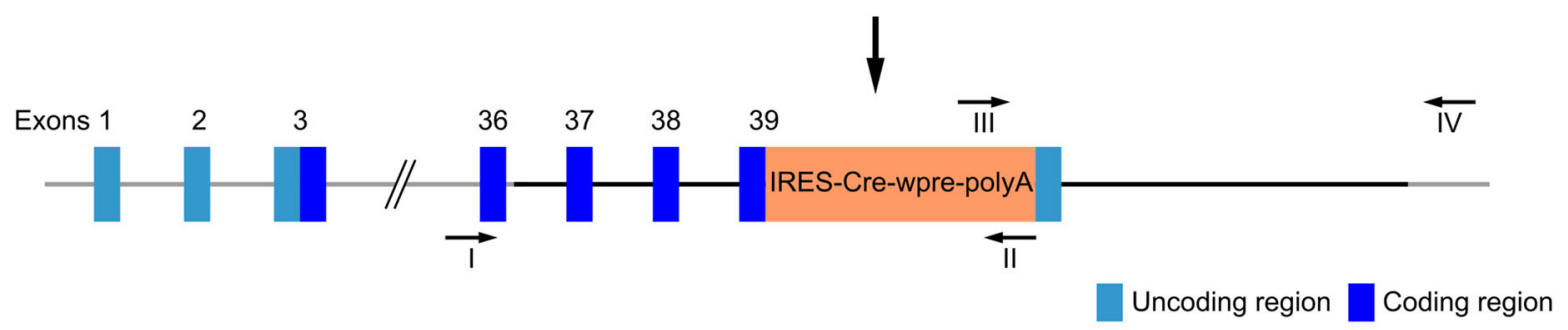

(b)
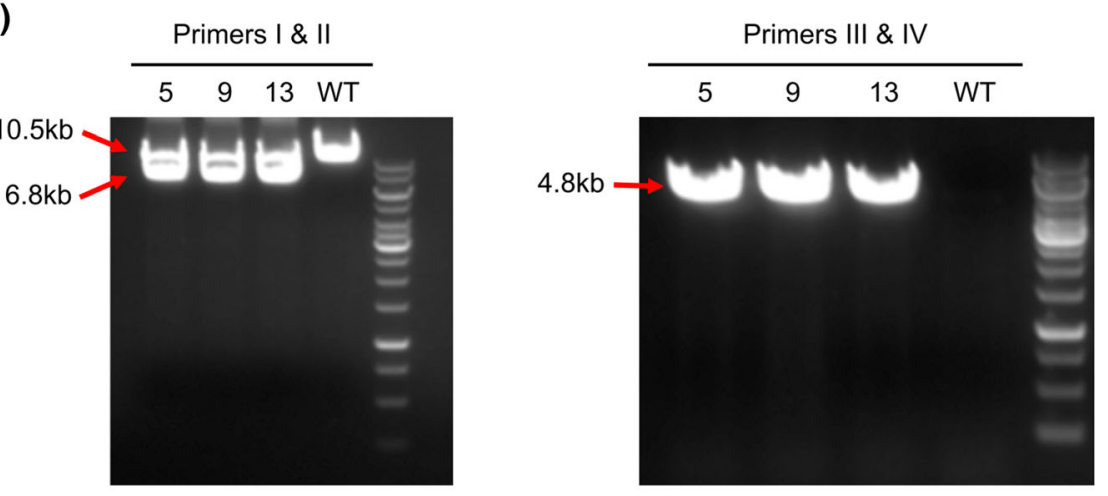

(c)

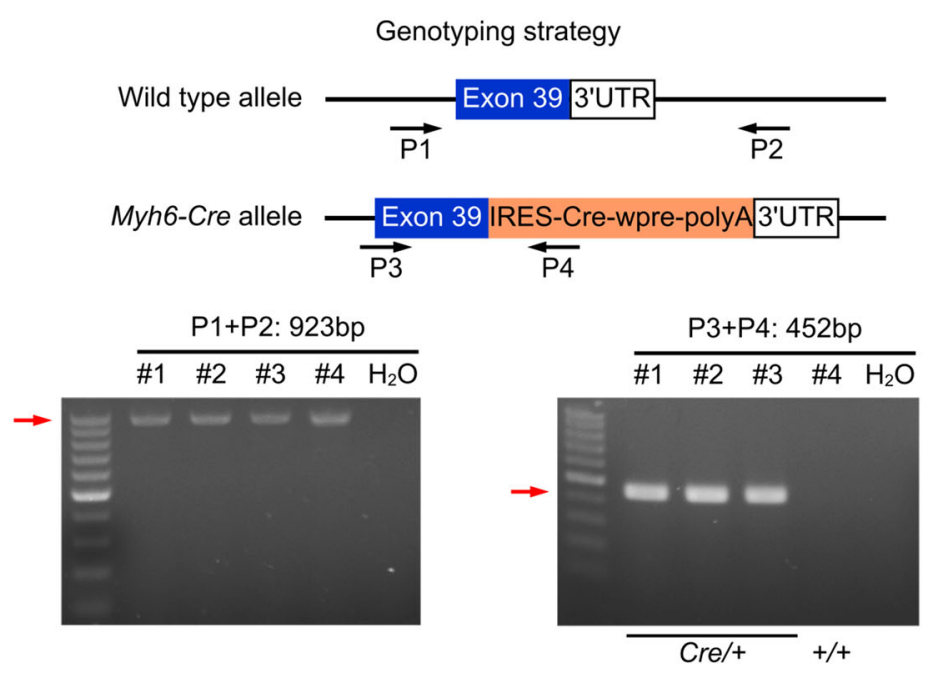


(a)

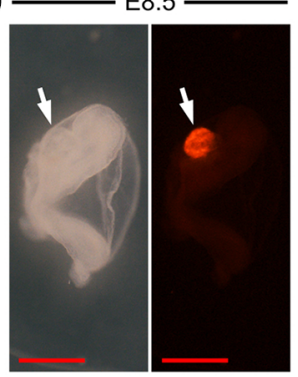

(c)

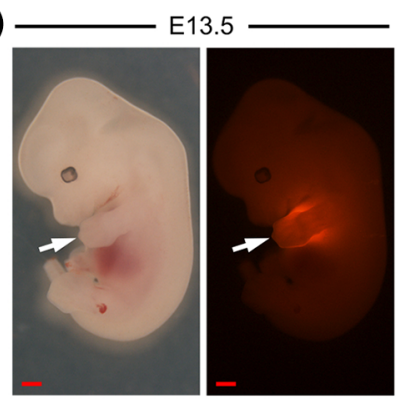

(e)

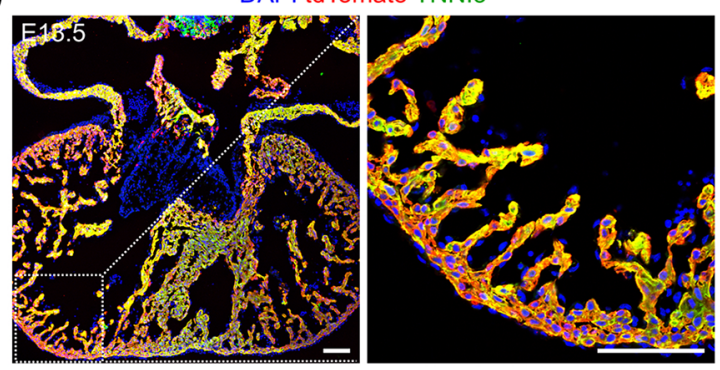

(f)

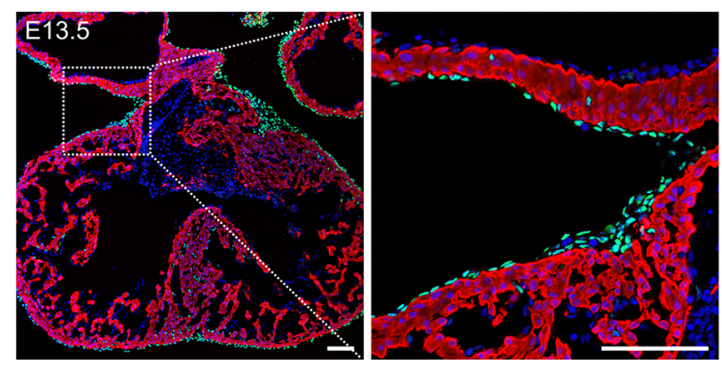

(g)

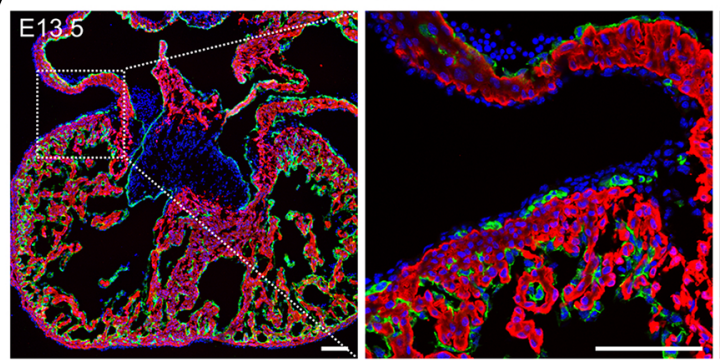

(d) (b)

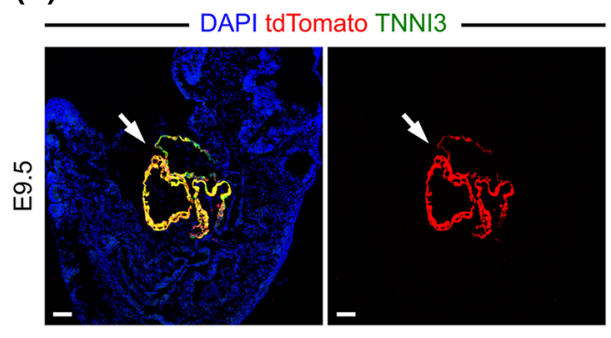

Stomach
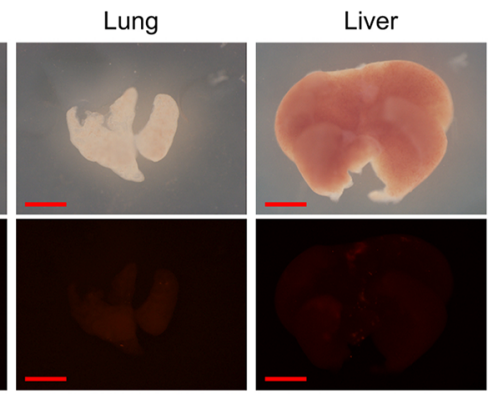

TNNI3

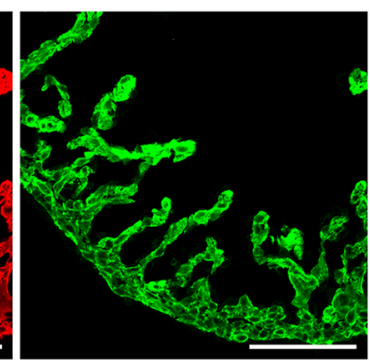

WT1
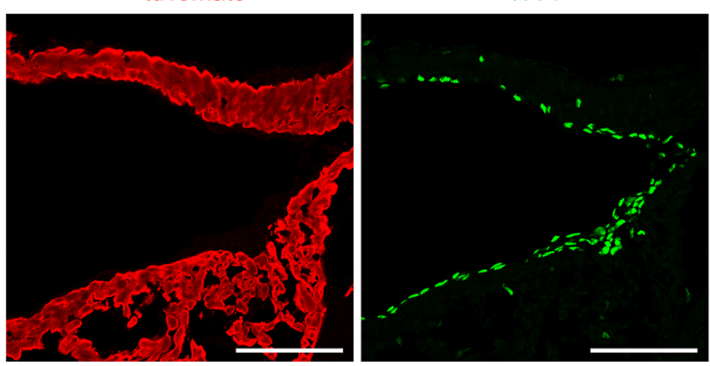

$\mathrm{CDH} 5$
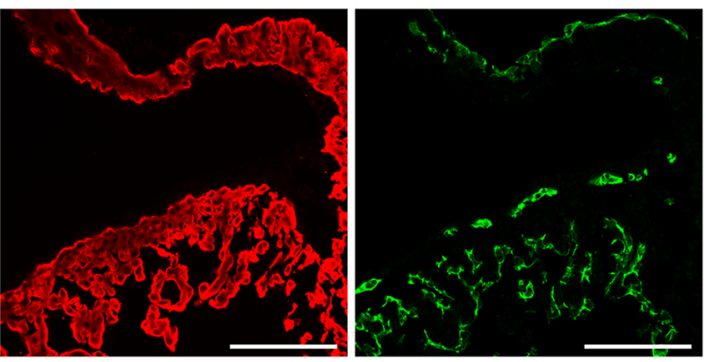
4Fig. 2 Myh6-Cre labels cardiomyocytes in embryonic hearts. (a, c) Whole-mount view of the Myh6-Cre/ $+; R 26$-tdTomato/ + embryos at E8.5 and E9.5. b Immunostaining for tdTomato and TNNI3 on embryonic sections at E9.5. The arrows in (ac) indicate hearts with enriched tdTomato signals. d Wholemount view of the organs from Myh6-Cre/ + ;R26-tdTomato/ + mice at E13.5. (e-g) Immunostaining for tdTomato and TNNI3, WT1 or CDH5 on heart sections from Myh6-Cre/ + ;R26-tdTomato/ + mice at E13.5. Boxed regions are magnified on the right. 4 embryos were examined for each embryonic stage. Red scale bars, $1 \mathrm{~mm}$; White scale bars, $100 \mu \mathrm{m}$

We examined the Myh6-Crel +;R26-tdTomato/ + embryos at different time points. At embryonic day (E) 8.5, we specifically detected tdTomato signals in the heart tubes (Fig. 2a). At E9.5, we found similar results that tdTomato was exclusively expressed in the hearts (Fig. 2a). Immunostaining for tdTomato and cardiomyocyte marker TNNI3 showed that most cardiomyocytes were labelled by tdTomato at E9.5 (Fig. 2b). We then analyzed the embryos at E13.5. tdTomato signals were robustly detected in the heart, but not in other organs, such as the stomach, lung and liver (Fig. 2c, d). Immunostaining for tdTomato and TNNI3 confirmed that the majority of cardiomyocytes were tdTomato $^{+}$at E13.5 (Fig. 2e). Moreover, costaining assays of different heart sections with tdTomato and either WT1 or CDH5 antibodies showed that tdTomato did not target any epicardial or endothelial cells at E13.5 (Fig. 2f, g). We also stained the Myh6Crel + embryonic hearts with TNNI3, MYH6 and Cre antibodies and found co-localization of Cre and MYH6 in TNNI3 ${ }^{+}$cardiomyocytes (Supplementary Fig. S1), suggesting that Cre activity is specifically localized to $\mathrm{MYH6}^{+}$cardiomyocytes with high fidelity in Myh6-Cre/ + embryonic hearts.

Next, we proceeded to analyze the Myh6-Cre/ + ;R26-tdTomato/ + mice at postnatal stages. We harvested the hearts at postnatal 2 weeks (P2W) and were able to detect significant tdTomato signals in the whole-mount views (Fig. 3a, b). Similarly, co-staining for tdTomato and TNNI3 on heart sections also demonstrated that the majority of cardiomyocytes were tdTomato $^{+}$at P2W (Fig. 3c). PDGFRa has been previously used as a marker for cardiac fibroblasts (Kaur et al. 2016; Huang et al. 2019; Feng et al. 2019).
Hence, in order to examine whether the lineage tracer marks cardiac fibroblasts at $\mathrm{P} 2 \mathrm{~W}$, we stained the heart sections with tdTomato and PDGFRa antibodies. No tdTomato-labelled $\mathrm{PDGFRa}^{+}$cells were found (Fig. 3d), suggesting that cardiac fibroblasts were not targeted by tdTomato. Additionally, we stained the hearts with antibodies against tdTomato, CDH5 and PDGFRb, a gene which was previously reported as a coronary vascular pericyte marker (Chen et al. 2016; Huang et al. 2019). Similarly, we could not find any tdTomato-labelled coronary endothelial cells or pericytes at P2W (Fig. 3e). Finally, we were not able to identify any tdTomato-labelled smooth muscle cells (SMCs) $\left(\mathrm{aSMA}^{+}\right)$in hearts at P2W (Fig. 3f). Taken together, our results provide strong evidence that Myh6-Crel + ;R26-tdTomato/ + specifically targets cardiomyocytes in early mouse infants.

We subsequently examined the hearts from adult Myh6-Crel + ;R26-tdTomatol + mice at P8W. Similar to $\mathrm{P} 2 \mathrm{~W}$, we identified strong tdTomato signals in the whole-mount view of the hearts (Fig. 4a, b), and found that the significant majority of cardiomyocytes were targeted by tdTomato by staining on heart sections (Fig. 4c). We also confirmed the tdTomato expression in the isolated cardiomyocytes from P8W hearts (Supplementary Fig. S2). Moreover, our observations confirmed that no $\mathrm{PDGFRa}^{+}$fibroblasts, $\mathrm{CDH}^{+}$coronary endothelial cells, or $\mathrm{PDGFRb}^{+}$ vascular pericytes were marked by tdTomato in the adult hearts (Fig. 4d, e). However, we did find tdTomato expression in a few coronary vascular SMCs (Fig. 4f). Microscopic quantification shows that $9.42 \pm 2.84 \%$ of SMCs are labeled in the adult Myh6-Crel + ;R26-tdTomatol + mouse hearts. Collectively, these observations demonstrate that Myh6Crel $+; R 26$-tdTomato/ + is also able to efficiently target cardiomyocytes in adult hearts.

To compare the labeling specificity Myh6-Cre with other similar Cre strains, we employed the inducible transgenic mouse line Myh6-MerCreMer that has been reported previously to target cardiomyocytes (Sohal et al. 2001). We administered the Myh6-MerCreMer $+;$ R26-tdTomato/ + mice with a dose of tamoxifen $(0.2 \mathrm{mg} / \mathrm{g}$ of body weight $)$ at P8W and harvested the hearts for analyses after $48 \mathrm{~h}$. tdTomato were 
(a)

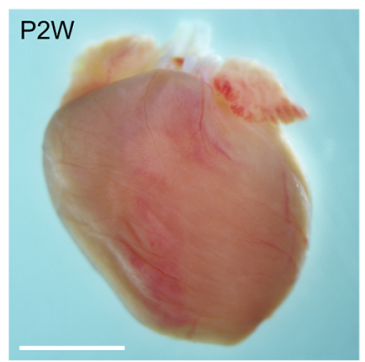

(c)

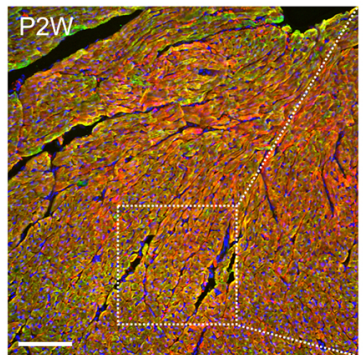

(d)

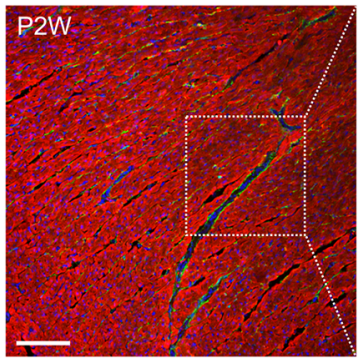

(e) $\mathrm{CDH} 5$ tdTomato PDGFRb

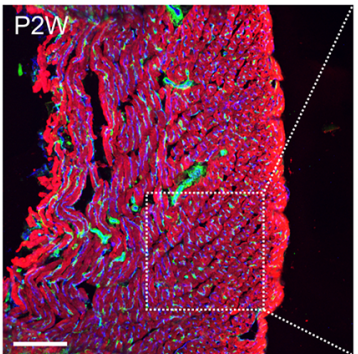

(f)

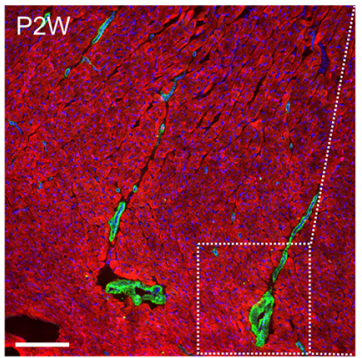

DAPI tdTomato TNNI3

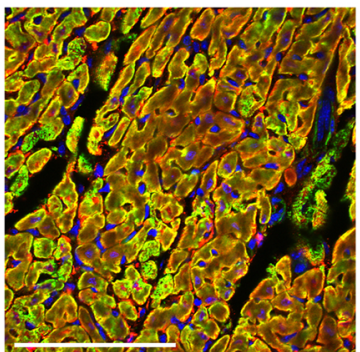

DAPI tdTomato PDGFRa

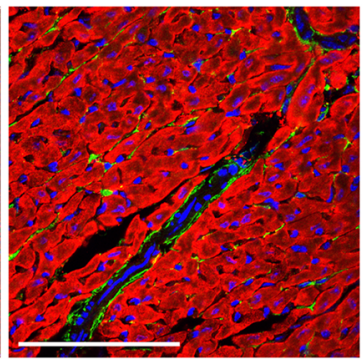

CDH5 tdTomato PDGFRb

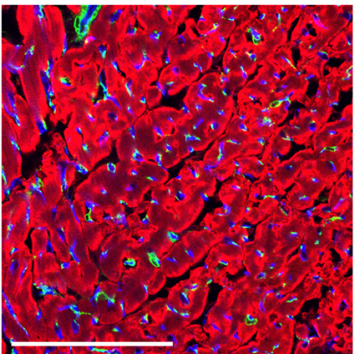

CDH5 tdTomato aSMA

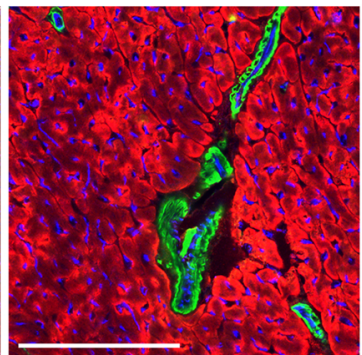

(b) P2W

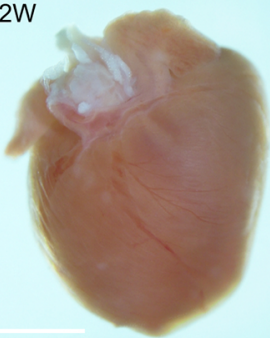

TNNI3

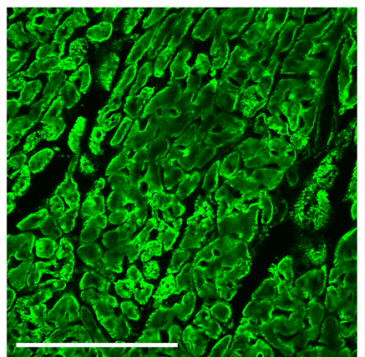

PDGFRa

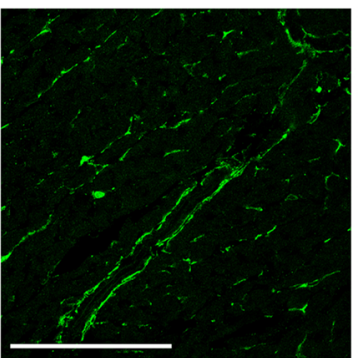

CDH5 PDGFRb

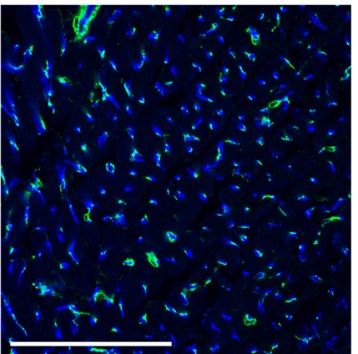

CDH5 aSMA

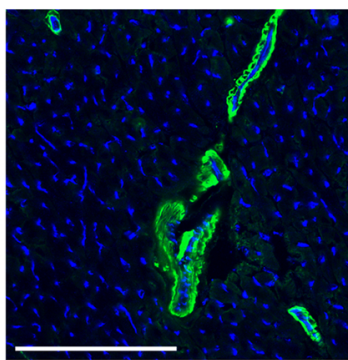

Dorsal

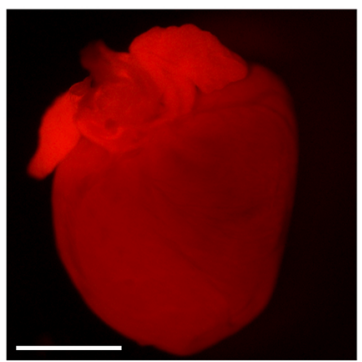

tdTomato

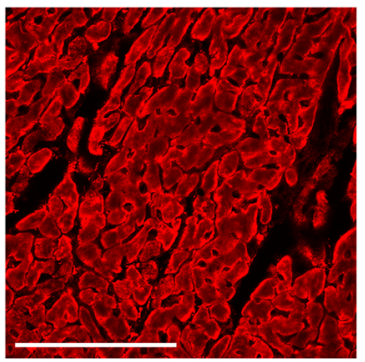

tdTomato

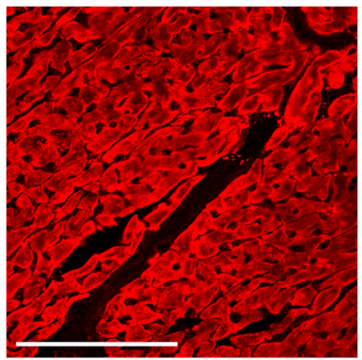

CDH5 tdTomato

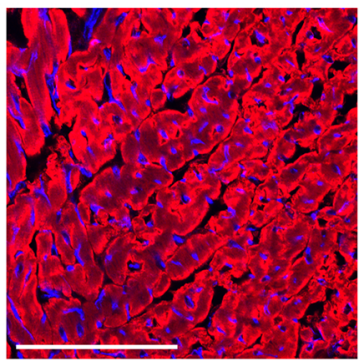

CDH5 tdTomato

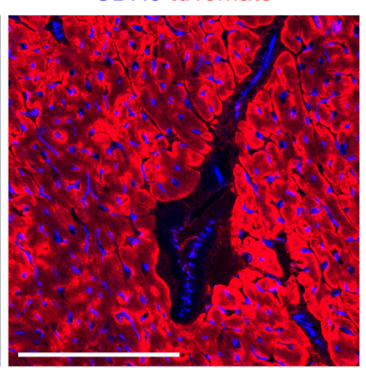


4Fig. 3 Myh6-Cre labels cardiomyocytes in infant mice. (a, b) Whole-mount view of the hearts from Myh6-Crel $+; R 26$ tdTomato/ + mice at P2W. Scale bars, $5 \mathrm{~mm}$. (c, d) Immunostaining for tdTomato and TNNI3 or PDGFRa on heart sections from Myh6-Crel + ;R26-tdTomato/ + mice at P2W. (e, f) Immunostaining for tdTomato, CDH5 and PDGFRb or aSMA on heart sections from Myh6-Crel + ;R26-tdTomatol + mice at $\mathrm{P} 2 \mathrm{~W}$. Boxed regions in $(\mathbf{c}-\mathbf{f})$ are magnified on the right. Scale bars in $(\mathbf{c}-\mathbf{f}), 100 \mu \mathrm{m}$. Each picture is representative of 3 individual mouse samples

detected in cardiomyocytes, but not in cardiac fibroblasts, pericytes, endothelial cells, or SMCs (Fig. 4g). We noted that the intensity of tdTomato signals in cardiomyocytes were remarkably inhomogeneous; and the tdTomato signals in the cytoplasm were too weak to be identified in a significant part of cardiomyocytes (Fig. 4g). Therefore, the specificity of Myh6MerCreMer in adult heart seems better than Myh6-Cre in this study, as Myh6-Cre is constitutively activated and also targets a few SMCs in adult hearts.

Next, we examined whether Myh6-Crel + ;R26tdTomato/ + targets other tissues or organs outside the heart at P8W. Few tdTomato signals were detected in the liver or thymus, but plenty of tdTomato ${ }^{+}$cells were identified in the kidney, spleen, skeletal muscle, and lung (Fig. 5a), suggesting the Cre expression in these non-cardiac tissues.

Myh6-Cre affects the expression of endogenous Myh6 in heart

Myh6-Cre strain can be maintained in the homozygous background and were also viable and fertile. However, we performed echocardiographic analysis at P8W and found that the heart functions of Myh6-Cre/Cre mice were compromised compared to wild type and Myh6Crel + groups (Supplementary Fig. S3, Fig. 5b). For instance, the left ventricular end-systolic dimension, left ventricular end-diastolic dimension, end-systolic volume, and end-diastolic volume were increased; and the ejection fraction and fractional shortening were decreased (Fig. 5b). There was no significant difference in heart function between the wild type and Myh6-Crel + groups at P8W (Supplementary Fig. S3, Fig. 5b). The appearance of Myh6-Cre/Cre mice was normal (Fig. 5c), but the hearts were hypertrophic (Fig. 5d, e). Histological examination showed thinner ventricular septa and left ventricular walls in the hearts of Myh6-Cre/Cre mice (Fig. 5f). Wheat germ agglutinin (WGA) staining showed the cardiomyocyte size significantly increased in the hearts of Myh6-Cre/Cre mice (Fig. 5g). These data suggest that the homozygous Myh6-Cre strain exhibits cardiac hypertrophy at the adult stage. We also examined heart functions of Myh6-Crel + mice at the later stage, and did not found any significant difference between the wild type and Myh6-Crel + groups at postnatal 4 months (Supplementary Fig. S4).

Because the homozygous Myh6-Cre mice had deteriorative heart function, we speculated that the insertion of IRES-Cre-wpre-poly A cassette between the translational stop codon and the $3^{\prime}$ UTR may affect the expression of endogenous Myh6 gene. Therefore, we performed real-time quantitative PCR and found that the expression level of Myh6 was decreased in the hearts of adult Myh6-Cre/Cre mice compared with the wild type and Myh6-Cre/ + groups (Fig. 5h). At the same time, the expression level of $\mathrm{Cre}$ was increased in the hearts of adult Myh6-Cre/Cre mice (Supplementary Fig. S5a). Western blot confirmed a reduced MYH6 protein level and increased Cre protein level in the hearts of adult Myh6-Cre/Cre mice (Supplementary Fig. S5b). The reason why Myh6 expression is downregulated by the insertion needs further investigation.

Deletion of desmoplakin gene in cardiomyocytes using Myh6-Cre

Desmoplakin (Dsp) is one of the members of the desmosome gene family. To further examine the deletion efficiency of Myh6-Cre, we crossed Dsp flox $\left(D s p^{f}\right)$ (Garcia-Gras et al. 2006) with Myh6-Cre mice in order to delete the Dsp gene in cardiomyocytes (Fig. 6a). We investigated whether Myh6-Cre was able to efficiently delete the Dsp gene in mouse hearts by crossing Myh6-Crel + ;Dsp $p^{f /+}$ with $D s p^{f / f l}$ mice. After this, we collected the embryos at E10.5 and performed real-time quantitative PCR experiments (primer P5 and P6 to amplify exon 2) to examine the mRNA levels of Dsp in the hearts (Fig. 6a). We found that $D s p$ mRNA was decreased in the hearts of mutant mice (Myh6-Crel + ;Dsp ${ }^{f / f l}$ ) compared to their littermate controls (Fig. 6b). We also analyzed the $D s p$ mRNA in the embryos without hearts at E10.5, and did not find any significant difference between the mutant and control groups (Fig. 6b). These data suggested 
(a)

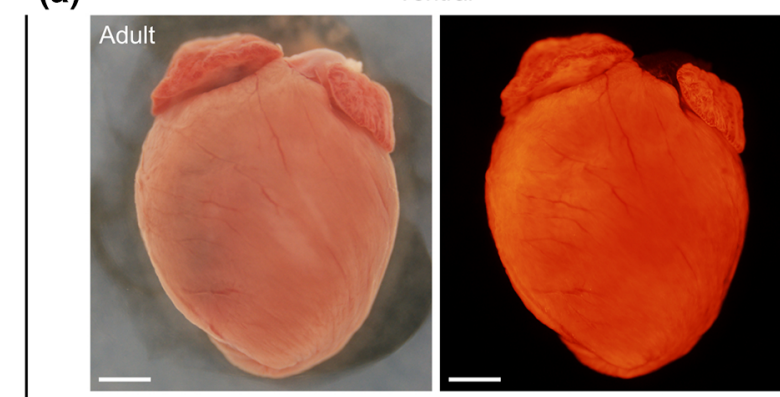

(c)

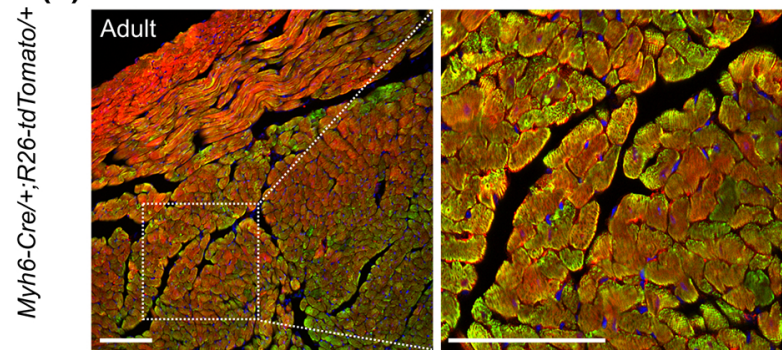

(e)
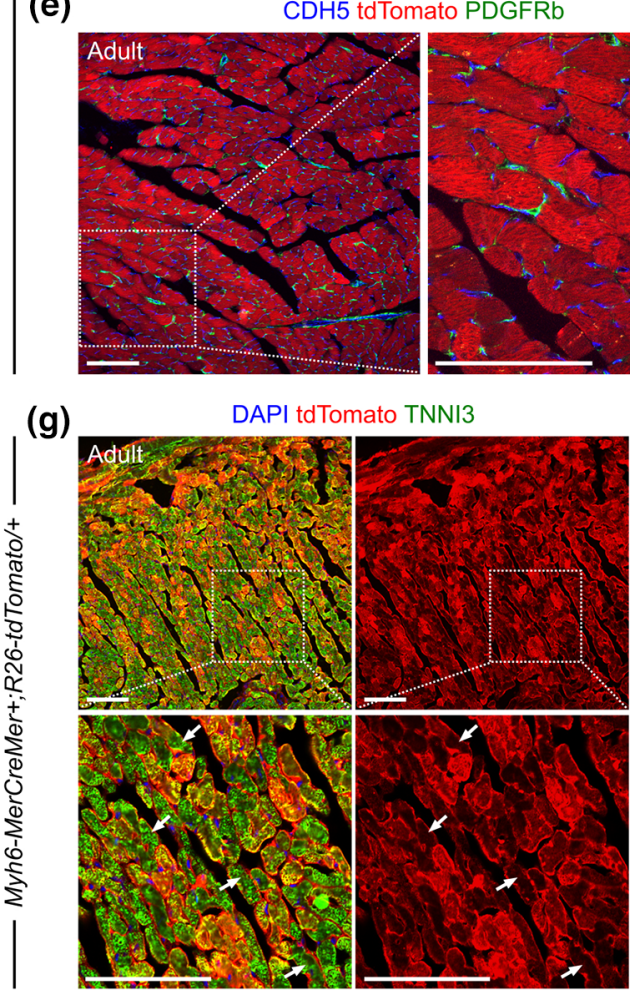

(b)

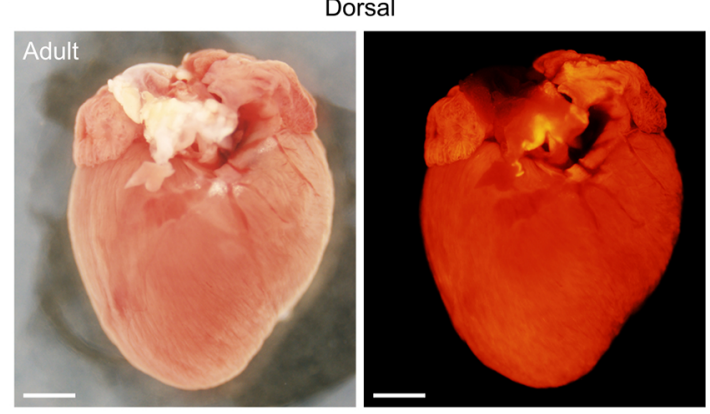

(d)

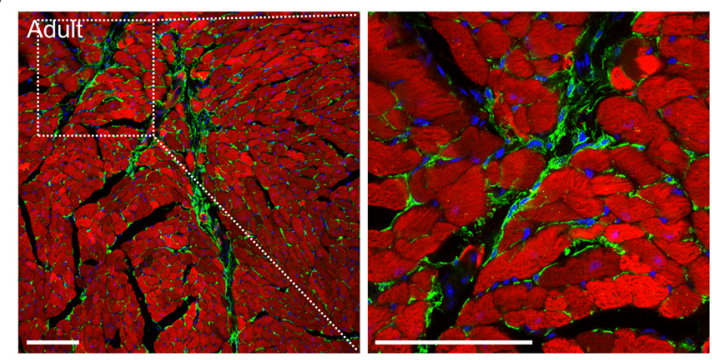

(f)

CDH5 tdTomato aSMA
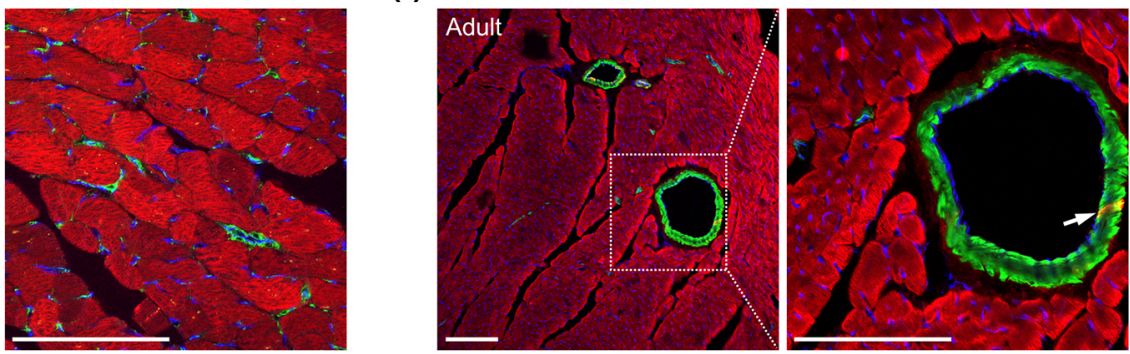

DAPI tdTomato PDGFRa $\quad$ CDH5 tdTomato PDGFRb CDH5 tdTomato aSMA
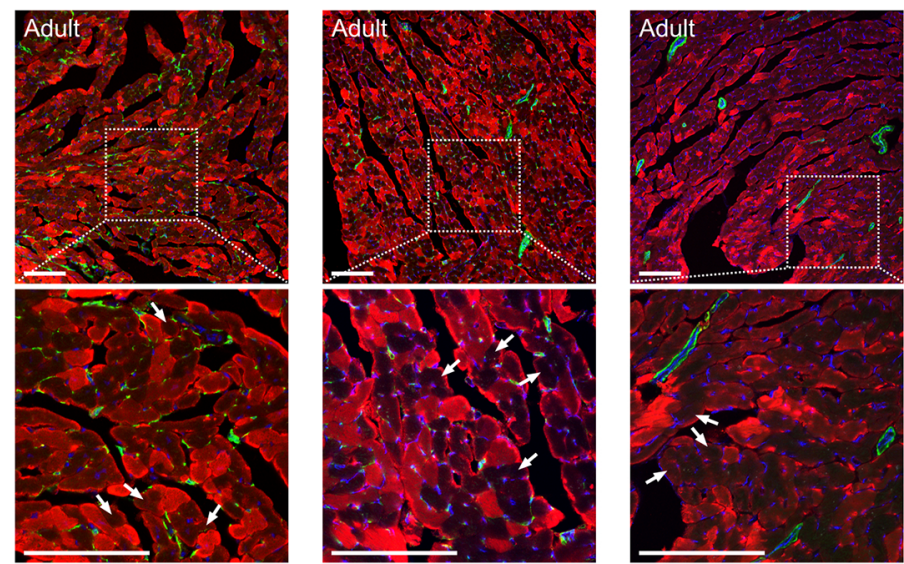
4Fig. 4 Myh6-Cre labels cardiomyocytes and a small portion of smooth muscle cells in adult hearts. (a, b) Whole-mount view of the hearts from adult Myh6-Crel $+;$ R26-tdTomato/ + mice. Scale bars, $2 \mathrm{~mm}$. (c, d) Immunostaining for tdTomato and TNNI3 or PDGFRa on heart sections from adult Myh6-Cre/ $+;$ R26-tdTomato/ + mice. (e, f) Immunostaining for tdTomato, CDH5 and PDGFRb or aSMA on heart sections from adult Myh6-Crel + ;R26-tdTomato/ + mice. The arrows in (f) indicate tdTomato-labelled smooth muscle cell. g Immunostaining on heart sections from adult Myh6-MerCreMer $+; R 26-$ tdTomato/ + mice, which were administered with tamoxifen $48 \mathrm{~h}$ before harvest. The arrows in ( $\mathbf{g}$ ) indicate cardiomyocytes with weak tdTomato signal in the cytoplasm. Boxed regions are magnified as indicated. Scale bars in $(\mathbf{c}-\mathbf{g}), 100 \mu \mathrm{m}$. Each picture is representative of 3 individual mouse samples

that the exon 2 of the Dsp gene was significantly deleted in the cardiomyocytes of mutant embryos.

Finally, we harvested the embryos at E11.5 and found that the morphology of the mutants was roughly normal compared to the controls (Fig. 6c). While at E13.5, the mutants appeared very pale and exhibited growth arrest at $\sim$ E11.5. The mutant hearts did not beat and were significantly smaller than those of littermate controls at E13.5 (Fig. 6c), suggesting that the inactivation of the $D s p$ gene in the cardiomyocytes severely influences heart development and results in embryonic lethality during mid-term pregnancy.

\section{Discussion}

A few constitutively activated Cre strains using Myh6 promoter are commercially available, such as $\mathrm{Tg}(\mathrm{My}-$ h6-Cre) (JAX stock \#009,074) (Oka et al. 2006) and Tg(Myh6-Cre) (JAX stock \#011,038) (Agah et al. 1997). These strains are transgenic and transgenes may not always correctly reflect the expression of endogenous Myh6 temporally or spatially. For instance, the transgenic Myh6-Cre line was reported to begin excising at E9.5 (Agah et al. 1997; Gaussin et al. 2002; Xu et al. 2009; Papanicolaou et al. 2012), while the knockin line Myh6-Cre we generated in this study turns on as early as E8.5. Therefore, this Myh6Cre knockin mouse line can be used to target cardiomyocytes since an earlier embryonic stage.

The Myh6-Cre in this study also labels a few SMCs in addition to cardiomyocytes in adult hearts. The inducible transgenic Myh6-MerCreMer strain (Sohal et al. 2001) is more specific in adult hearts likely because the MerCreMer allele can be activated in the restricted temporal window, while our Myh6-Cre is constitutively activated. Dynamic expression of Cre in unpredicted tissues or cells are always the issue for every constitutive Cre line.

Dsp is one of desmosomal genes, which also include desmocllin-2, desmoglein-2, plakophilin-2, and plakoglobin. Previous studies have identified mutations in all of the known desmosomal genes in patients with arrhythmogenic cardiomyopathy (Austin et al. 2019). Early embryonic lethality has been reported in the germline Dsp-null mice (Gallicano et al. 1998). Deletion of the Dsp gene in cardiomyocytes using transgenic $\alpha M H C$-Cre mice also leads to high lethality in embryos (Garcia-Gras et al. 2006). The cardiac-restricted Dsp mutants exhibited growth arrest at E10-E12, and the specific phenotypes of the mutants have been investigated in more detail in the previous study (Garcia-Gras et al. 2006). In this study, just to examine the deletion efficiency of Myh6-Cre, we crossed the $D s p^{f l}$ mice with Myh6-Cre mice and found that the exon 2 of the Dsp gene was significantly removed in the E10.5 hearts by the Myh6-Cre. The Dsp mutants in this study exhibited growth arrest at $\sim$ E11.5, in agreement with the previous observations (Garcia-Gras et al. 2006).

In summary, we established a new Myh6-Cre mouse model as an efficient tool enabling targeted gene deletion in cardiomyocytes. This model may thus significantly improve our knowledge and address critical questions regarding heart development and diseases. 
(a)
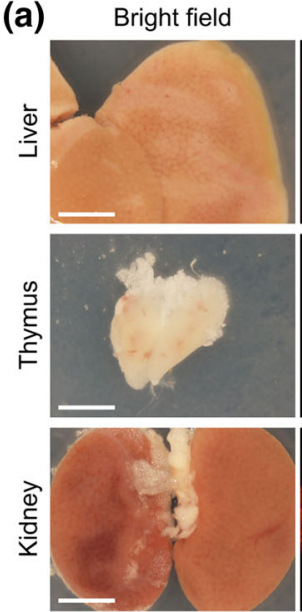

Fluorescent
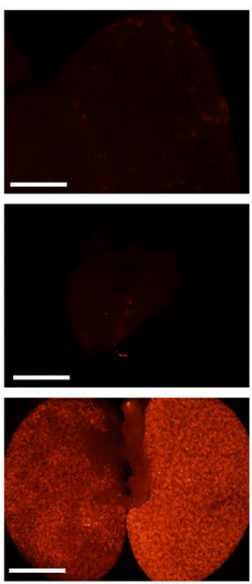

DAPI tdTomato
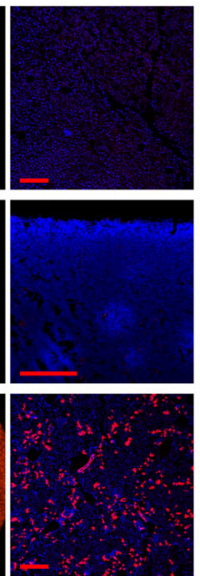

(b)

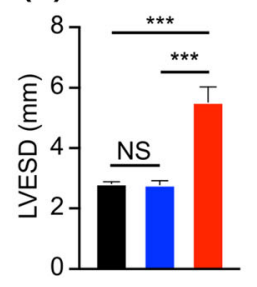

Myh6 +/+

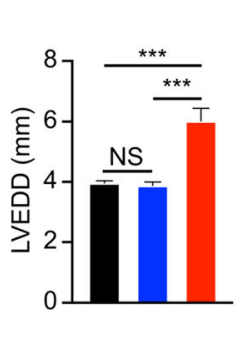

Myh6-Cre/+
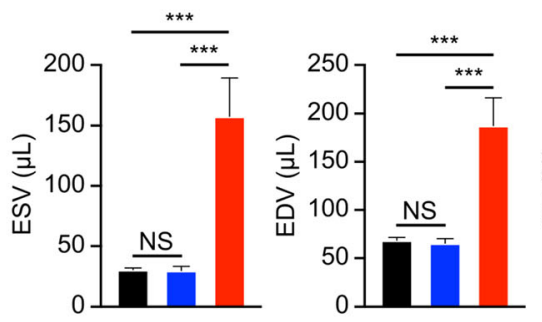

DAPI tdTomato
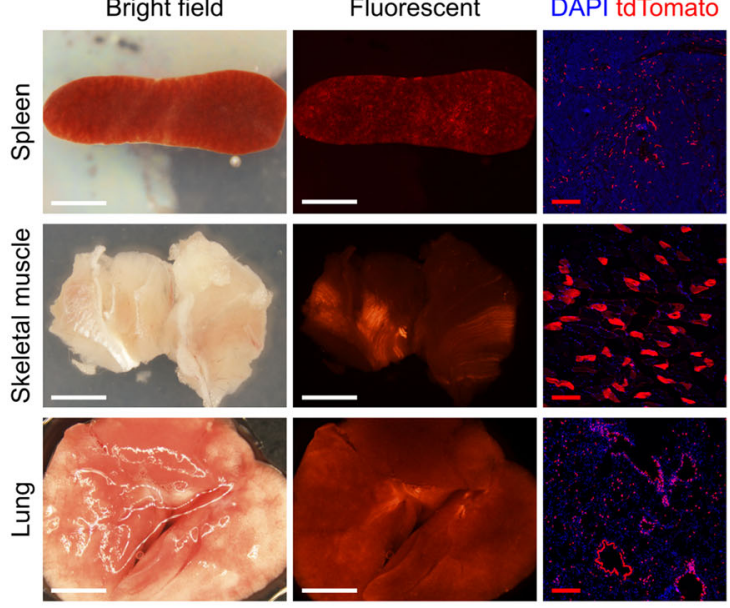

(c)

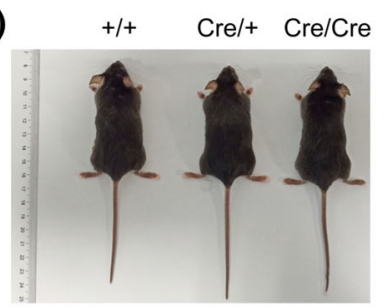

(d)

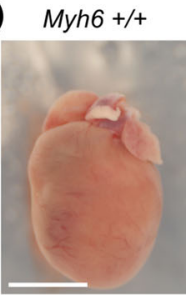

Myh6-Cre/+

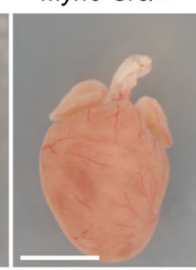

Myh6-Cre/Cre

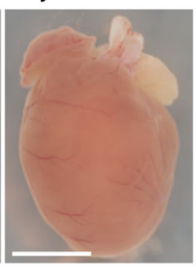

(e)

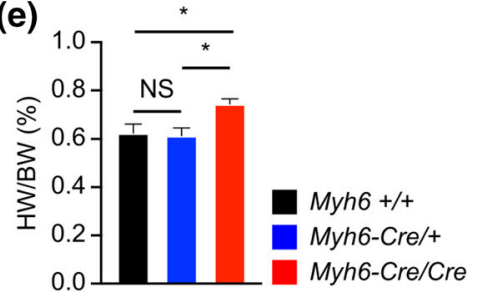

(f)

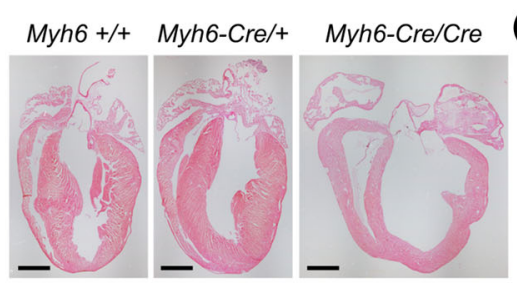

(g)

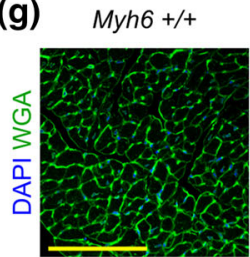

Myh6-Cre/+

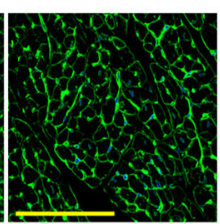

Myh6-Cre/Cre

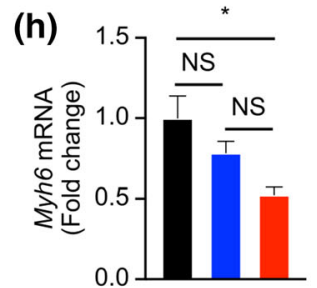

Fig. 5 Myh6-Cre targets some other organs outside the heart and affects the expression of endogenous Myh6 in heart. a tdTomato was detected in some other organs from the adult Myh6-Crel + ;R26-tdTomato/ + mice at P8W. 3 mouse samples were examined. b ECG analyses of the P8W hearts with indicated genotypes. $n=6$ mice per group. LVESD, left ventricular end-systolic dimension; LVEDD, left ventricular end-diastolic dimension; ESV, end-systolic volume; EDV, enddiastolic volume; EF, ejection fraction; FS, fractional shortening. c Whole bodies of the mice with indicated genotypes at
P8W. d Whole-mount view of the hearts with indicated genotypes at P8W. e The relative heart weight was increased in the Myh6-Cre/Cre mice at P8W. HW, heart weight. BW, body weight. f HE staining shows the thinner left ventricular wall and ventricular septum in the hearts of Myh6-Cre/Cre mice at P8W. g WGA staining on heart sections. $\mathbf{h}$ The expression level of Myh6 in the adult hearts at P8W. $n=3$ mice per group in $(\mathbf{c}-\mathbf{h})$. White scale bars, $5 \mathrm{~mm}$; Black scale bars, $2 \mathrm{~mm}$; Red scale bars, $200 \mu \mathrm{m}$; Yellow scale bars, $100 \mu \mathrm{m}$. NS, non-significant; $* p<0.05 ; * * * p<0.001$ 
(a)

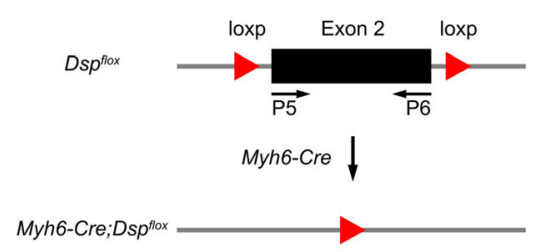

(b)

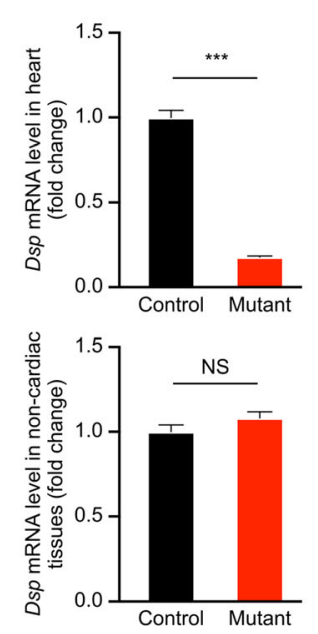

Fig. 6 Inactivation of the $D s p$ gene in cardiomyocytes using Myh6-Cre. a The strategy for inactivating the Dsp gene in cardiomyocytes using Myh6-Cre. Primers P5 and P6 were used to detect exon 2. b The relative expression level of $D s p$ mRNA

Acknowledgements This work was sponsored by grants from National Natural Science Foundation of China (31822034, 31871474, 81861128023, 81970328), the National key R\&D Program of China (2018YFA0108100), the "Shuguang Program" supported by Shanghai Education Development Foundation and Shanghai Municipal Commission (17SG54), Shanghai Rising-Star Program (20QA1406900), and the ShanghaiTech University start-up fund. We thank the Molecular Imaging Core Facility (MICF) and the Molecular and Cell Biology Core Facility (MCBCF) at the School of Life Science and Technology, ShanghaiTech University for providing technical support. We thank Haojie Chen, Chaohua Zheng, Hao Feng, and Jianjie Gu for the animal husbandry.

Authors' contributions Xinyan Huang, Lei Yan, Chen Liu and Hui Zhang contributed to the study conception and design. Material preparation, data collection and analyses were performed by Xinyan Huang, Lei Yan, Shan Kou, Jufeng Meng and Zhengkai Lu. The manuscript was written by Xinyan Huang, Lei Yan and Hui Zhang. Chao-Po Lin and Chen Liu edited and commented the manuscript.

Data availability All data generated and analyzed during this study are included in this published article. (c)
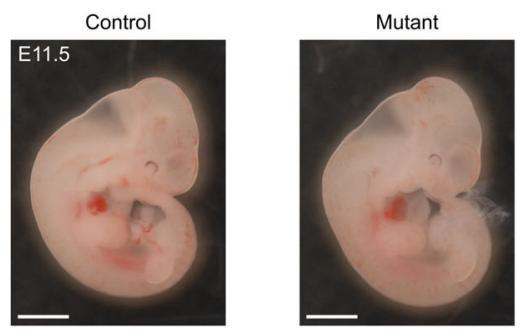

(d)
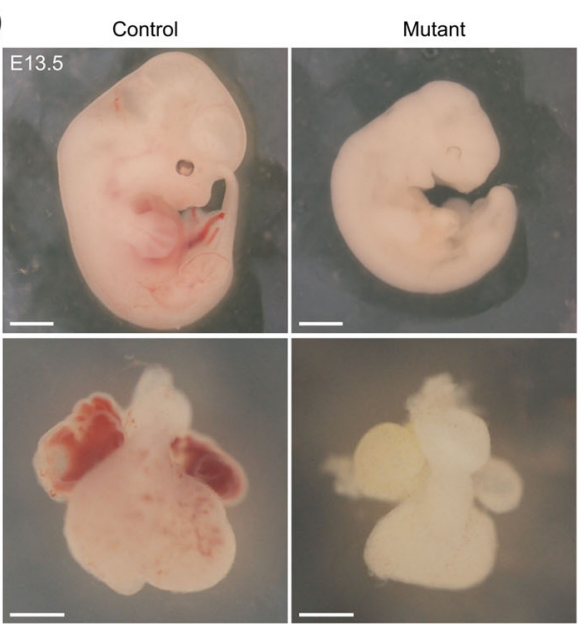

in hearts or non-cardiac tissues at E10.5. *** $p<0.001$; NS, non-significant. $n=3$ hearts per group. (c, d) Whole-mount view of embryos and hearts at E11.5 and E13.5. $n=4$ embryos per group. Scale bars, $2 \mathrm{~mm}$

\section{Declarations}

Conflict of interest The authors declare no conflicts of interest in this work.

Ethical approval All animal experiments were approval by the institutional Animal Care and Use Committee at ShanghaiTech University.

Consent to participate Informed consent was obtained from all individual participants included in the study.

Consent for publication The participants have consented to the submission of this study to the journal.

Open Access This article is licensed under a Creative Commons Attribution 4.0 International License, which permits use, sharing, adaptation, distribution and reproduction in any medium or format, as long as you give appropriate credit to the original author(s) and the source, provide a link to the Creative Commons licence, and indicate if changes were made. The images or other third party material in this article are included in the article's Creative Commons licence, unless indicated otherwise in a credit line to the material. If material is not included in the article's Creative Commons licence and your intended use is not permitted by statutory regulation or exceeds 
the permitted use, you will need to obtain permission directly from the copyright holder. To view a copy of this licence, visit http://creativecommons.org/licenses/by/4.0/.

\section{References}

Agah R, Frenkel PA, French BA, Michael LH, Overbeek PA, Schneider MD (1997) Gene recombination in postmitotic cells. Targeted expression of Cre recombinase provokes cardiac-restricted, site-specific rearrangement in adult ventricular muscle in vivo. J Clin Invest 100(1):169-179. https://doi.org/10.1172/JCI119509

Austin KM, Trembley MA, Chandler SF, Sanders SP, Saffitz JE, Abrams DJ, Pu WT (2019) Molecular mechanisms of arrhythmogenic cardiomyopathy. Nat Rev Cardiol 16(9):519-537. https://doi.org/10.1038/s41569-019-02007

Bersell K, Choudhury S, Mollova M, Polizzotti BD, Ganapathy B, Walsh S, Wadugu B, Arab S, Kuhn B (2013) Moderate and high amounts of tamoxifen in alphaMHC-MerCreMer mice induce a DNA damage response, leading to heart failure and death. Dis Model Mech 6(6):1459-1469. https://doi.org/10.1242/dmm.010447

Bruneau BG, Srivastava D (2014) Congenital heart disease: entering a new era of human genetics. Circ Res 114(4):598-599. https://doi.org/10.1161/CIRCRESAHA. 113.303060

Buerger A, Rozhitskaya O, Sherwood MC, Dorfman AL, Bisping E, Abel ED, Pu WT, Izumo S, Jay PY (2006) Dilated cardiomyopathy resulting from high-level myocardial expression of Cre-recombinase. J Card Fail 12(5):392-398. https://doi.org/10.1016/j.cardfail.2006.03. 002

Carniel E, Taylor MR, Sinagra G, Di Lenarda A, Ku L, Fain PR, Boucek MM, Cavanaugh J, Miocic S, Slavov D, Graw SL, Feiger J, Zhu XZ, Dao D, Ferguson DA, Bristow MR, Mestroni L (2005) Alpha-myosin heavy chain: a sarcomeric gene associated with dilated and hypertrophic phenotypes of cardiomyopathy. Circulation 112(1):54-59. https://doi.org/10.1161/CIRCULATIONAHA.104.507699

Chen Q, Zhang H, Liu Y, Adams S, Eilken H, Stehling M, Corada M, Dejana E, Zhou B, Adams RH (2016) Endothelial cells are progenitors of cardiac pericytes and vascular smooth muscle cells. Nat Commun 7:12422. https://doi.org/10.1038/ncomms12422

Ching YH, Ghosh TK, Cross SJ, Packham EA, Honeyman L, Loughna S, Robinson TE, Dearlove AM, Ribas G, Bonser AJ, Thomas NR, Scotter AJ, Caves LS, Tyrrell GP, Newbury-Ecob RA, Munnich A, Bonnet D, Brook JD (2005) Mutation in myosin heavy chain 6 causes atrial septal defect. Nat Genet 37(4):423-428. https://doi.org/10.1038/ ng 1526

Davis J, Maillet M, Miano JM, Molkentin JD (2012) Lost in transgenesis: a user's guide for genetically manipulating the mouse in cardiac research. Circ Res 111(6):761-777. https://doi.org/10.1161/CIRCRESAHA.111.262717
Feng T, Meng J, Kou S, Jiang Z, Huang X, Lu Z, Zhao H, Lau LF, Zhou B, Zhang H (2019) CCN1-induced cellular senescence promotes heart regeneration. Circulation 139(21):2495-2498. CIRCULATIONAHA.119.039530

Gallicano GI, Kouklis P, Bauer C, Yin M, Vasioukhin V, Degenstein L, Fuchs E (1998) Desmoplakin is required early in development for assembly of desmosomes and cytoskeletal linkage. J Cell Biol 143(7):2009-2022. https:// doi.org/10.1083/jcb.143.7.2009

Garcia-Gras E, Lombardi R, Giocondo MJ, Willerson JT, Schneider MD, Khoury DS, Marian AJ (2006) Suppression of canonical Wnt/beta-catenin signaling by nuclear plakoglobin recapitulates phenotype of arrhythmogenic right ventricular cardiomyopathy. J Clin Invest 116(7):2012-2021. https://doi.org/10.1172/JCI27751

Gaussin V, Van de Putte T, Mishina Y, Hanks MC, Zwijsen A, Huylebroeck D, Behringer RR, Schneider MD (2002) Endocardial cushion and myocardial defects after cardiac myocyte-specific conditional deletion of the bone morphogenetic protein receptor ALK3. Proc Natl Acad Sci U S A 99(5):2878-2883. https://doi.org/10.1073/pnas. 042390499

Gelb BD, Chung WK (2014) Complex genetics and the etiology of human congenital heart disease. Cold Spring Harb Perspect Med 4(7):a013953. https://doi.org/10.1101/ cshperspect.a013953

Hall ME, Smith G, Hall JE, Stec DE (2011) Systolic dysfunction in cardiac-specific ligand-inducible MerCreMer transgenic mice. Am J Physiol Heart Circ Physiol 301(1):H253-260. https://doi.org/10.1152/ajpheart.00786.2010

Hoffman JI (1995) Incidence of congenital heart disease: II. Prenatal Incidence Pediatr Cardiol 16(4):155-165. https:// doi.org/10.1007/BF00794186

Hougen K, Aronsen JM, Stokke MK, Enger U, Nygard S, Andersson KB, Christensen G, Sejersted OM, Sjaastad I (2010) Cre-loxP DNA recombination is possible with only minimal unspecific transcriptional changes and without cardiomyopathy in $\mathrm{Tg}$ (alphaMHC-MerCreMer) mice. Am J Physiol Heart Circ Physiol 299(5):H1671-1678. https:// doi.org/10.1152/ajpheart.01155.2009

Huang X, Feng T, Jiang Z, Meng J, Kou S, Lu Z, Chen W, Lin CP, Zhou B, Zhang H (2019) Dual lineage tracing identifies intermediate mesenchymal stage for endocardial contribution to fibroblasts, coronary mural cells, and adipocytes. J Biol Chem 294(22):8894-8906. https://doi.org/10.1074/ jbc.RA118.006994

Kaur H, Takefuji M, Ngai CY, Carvalho J, Bayer J, Wietelmann A, Poetsch A, Hoelper S, Conway SJ, Mollmann H, Looso M, Troidl C, Offermanns S, Wettschureck N (2016) Targeted ablation of periostin-expressing activated fibroblasts prevents adverse cardiac remodeling in mice. Circ Res 118(12):1906-1917. https://doi.org/10.1161/ CIRCRESAHA.116.308643

Koitabashi N, Bedja D, Zaiman AL, Pinto YM, Zhang M, Gabrielson KL, Takimoto E, Kass DA (2009) Avoidance of transient cardiomyopathy in cardiomyocyte-targeted tamoxifen-induced MerCreMer gene deletion models. Circ Res 105(1):12-15. https://doi.org/10.1161/ CIRCRESAHA.109.198416 
Laboulaye MA, Duan X, Qiao M, Whitney IE, Sanes JR (2018) Mapping transgene insertion sites reveals complex interactions between mouse transgenes and neighboring endogenous genes. Front Mol Neurosci 11:385. https://doi. org/10.3389/fnmol.2018.00385

Lexow J, Poggioli T, Sarathchandra P, Santini MP, Rosenthal N (2013) Cardiac fibrosis in mice expressing an inducible myocardial-specific Cre driver. Dis Model Mech 6(6):1470-1476. https://doi.org/10.1242/dmm.010470

Madisen L, Zwingman TA, Sunkin SM, Oh SW, Zariwala HA, Gu H, Ng LL, Palmiter RD, Hawrylycz MJ, Jones AR, Lein ES, Zeng H (2010) A robust and high-throughput Cre reporting and characterization system for the whole mouse brain. Nat Neurosci 13(1):133-140. https://doi.org/10. 1038/nn.2467

Molkentin JD, Robbins J (2009) With great power comes great responsibility: using mouse genetics to study cardiac hypertrophy and failure. J Mol Cell Cardiol 46(2):130-136. https://doi.org/10.1016/j.yjmcc.2008.09.002

Ng WA, Grupp IL, Subramaniam A, Robbins J (1991) Cardiac myosin heavy chain mRNA expression and myocardial function in the mouse heart. Circ Res 68(6):1742-1750. https://doi.org/10.1161/01.res.68.6.1742

Oka T, Maillet M, Watt AJ, Schwartz RJ, Aronow BJ, Duncan SA, Molkentin JD (2006) Cardiac-specific deletion of Gata4 reveals its requirement for hypertrophy, compensation, and myocyte viability. Circ Res 98(6):837-845. https://doi.org/10.1161/01.RES.0000215985.18538.c4

Papanicolaou KN, Kikuchi R, Ngoh GA, Coughlan KA, Dominguez I, Stanley WC, Walsh K (2012) Mitofusins 1 and 2 are essential for postnatal metabolic remodeling in heart. Circ Res 111(8):1012-1026. https://doi.org/10.1161/ CIRCRESAHA.112.274142

Posch MG, Waldmuller S, Muller M, Scheffold T, Fournier D, Andrade-Navarro MA, De Geeter B, Guillaumont S, Dauphin C, Yousseff D, Schmitt KR, Perrot A, Berger F, Hetzer R, Bouvagnet P, Ozcelik C (2011) Cardiac alpha- myosin (MYH6) is the predominant sarcomeric disease gene for familial atrial septal defects. PLoS ONE 6(12):e28872. https://doi.org/10.1371/journal.pone. 0028872

Richard P, Charron P, Carrier L, Ledeuil C, Cheav T, Pichereau C, Benaiche A, Isnard R, Dubourg O, Burban M, Gueffet JP, Millaire A, Desnos M, Schwartz K, Hainque B, Komajda M, Project EHF (2003) Hypertrophic cardiomyopathy - Distribution of disease genes, spectrum of mutations, and implications for a molecular diagnosis strategy. Circulation 107(17):2227-2232. https://doi.org/10.1161/ 01.Cir.0000066323.15244.54

Sohal DS, Nghiem M, Crackower MA, Witt SA, Kimball TR, Tymitz KM, Penninger JM, Molkentin JD (2001) Temporally regulated and tissue-specific gene manipulations in the adult and embryonic heart using a tamoxifen-inducible Cre protein. Circ Res 89(1):20-25. https://doi.org/10.1161/ hh1301.092687

Vasioukhin V, Bowers E, Bauer C, Degenstein L, Fuchs E (2001) Desmoplakin is essential in epidermal sheet formation. Nat Cell Biol 3(12):1076-1085. https://doi.org/10. 1038/ncb1201-1076

Wolf M, Basson CT (2010) The molecular genetics of congenital heart disease: a review of recent developments. Curr Opin Cardiol 25(3):192-197. https://doi.org/10.1097/ HCO.0b013e328337b4ce

$\mathrm{Xu} \mathrm{J}$, Ismat FA, Wang T, Lu MM, Antonucci N, Epstein JA (2009) Cardiomyocyte-specific loss of neurofibromin promotes cardiac hypertrophy and dysfunction. Circ Res 105(3):304-311. https://doi.org/10.1161/CIRCRESAHA. 109.201509

Publisher's Note Springer Nature remains neutral with regard to jurisdictional claims in published maps and institutional affiliations. 